\title{
Control of Sheerness in Pantyhose Fabric
}

\author{
Hiroaki ISHIZAWA, Lina WAKAKO, Hiroyuki KANAI, Kazuo SHINOHARA, \\ Toyonori NISHIMATSU, Hirofusa SHIRAI, AND Yo-ichi MATSUMOTO
}

\author{
Faculty of Textile Science and Technology, Shinshu University, Ueda, Japan \\ Hideo MOROOKA \\ Department of Textile and Apparel Science, Nara Women's University, Nara, Japan
}

Received December 22, 2003; Accepted for Publication April 20, 2004

\begin{abstract}
To clarify the aesthetic properties of pantyhose fabric, the design and development of a new product and a wear experiment were carried out. The sheerness of circular plain knitted fabrics made from novel single covered yarns was examined. The relationships between the leg parts of pantyhose fabric with two covering levels in one single covered yarn and the visual impression are discussed. The results show that the aesthetics and sheerness of pantyhose fabric are greatly influenced by changing the covering level of the single covered yarn in the leg parts. It is important to decrease the mean and the standard deviation of the lightness difference between legs with and without the pantyhose fabric on the lower leg part from knee to ankle. A mechanical hybrid system with combined covering and knitting processes is useful for improving the aesthetic properties of pantyhose fabric.
\end{abstract}

Keywords: Pantyhose; Aesthetic appeal; Single covered yarn; Variation of lightness difference; Hybrid knitting system.

\section{Introduction}

The aesthetic properties of pantyhose have long been of great concern to women, even more so in recent years. The sheerness of pantyhose is one of the important aesthetic properties for a woman's leg [8, 10]. However, the appearance and sheerness of pantyhose currently on the market is not yet satisfactory to meet women's needs. Previous papers on the performance and comfort of pantyhose have been published by various authors $[2-4,6]$. On the other hand, studies on the sheerness of pantyhose are rare. Sheerness is greatly affected by transmittance of light, which cannot be defined simply by the single covered yarn count. The evenness of sheerness over the entire leg is influenced by the degree of pantyhose extension on each leg part and unevenness affects the appearance, because the circumference decreases from thigh to ankle. In previous papers [1, 9], we examined the relationship between the covering level of single covered yarns, transmittance of light, color, and degree of extension of knitted fabrics. The appearance and sheerness of pantyhose varied with the color and were improved by increasing the degree of transmittance of light, the degree of fabric extension, and the covering level of the single covered yarn. In addition, it was necessary to evaluate the light difference of a uniform color space for a colorless pantyhose fabric.
In production of pantyhose, knitting process is usually separated from yarn making process. It is very important to consider that the development and improvement of pantyhose is delayed by the separation. In this paper, we report on the two tests of the visual impressions and image analysis of the fronts of legs wearing pantyhose samples. And we consider a method of calculating their aesthetic properties in order to develop new pantyhose that can appeal the beauty of bare skin leg. We describe our progress in improving the aesthetic appeal by producing novel pantyhose with two covering levels in one single covered yarn using an experimental hybrid knitting system which combines the covering with the knitted processes [7].

\section{Materials and Methods}

\subsection{Samples}

We have produced an experimental hybrid knitting system for design and development of new pantyhose. This system is composed of two experimental covering machines and a circular knitting machine. Each covering machine has two sections of single covered yarn made for the knitting machine with four holes for supplying yarns. Single covered yarn is produced from a combination of polyurethane yarn ( 20 denier $/ 2$ filaments) as core yarn and nylon yarn (10 denier/7

* Corresponding author Fax:+81-268-21-5363, E-mail: y1matsu@giptc.shinshu-u.ac.jp 
filaments) as covering yarn. The setup conditions of the three main parts, i.e. the core tension, the speed of the hollow spindle, and the speed of the take-up roller affect the covering level of the single cover yarn produced. In addition, it is necessary to mesh both the speed for producing the single covered yarn and that of the machine knitting the pantyhose. In this hybrid system, pantyhose samples are knitted under a constant condition, while the single covered yarns are produced by controlling the covering levels of 1,500 tpm and 3,000 tpm in one yarn with the draw ratio of the core yarn $=2$. The covering level of $3,000 \mathrm{tpm}$ is chosen by the two following reasons: (1) The sheerness of pantyhose varies gradually from thigh to ankle and becomes less transparent farther down the leg; (2) The light transmittivity of the ankle part is about half of that of the knee part [9]. Namely, we try to bring the leg wearing pantyhose close to the bare skin leg as aesthetic leg. The resulting sample is a circular plain knitted fabric to cover the entire leg from thigh to toe. Table 1 lists the specifications of the colorless pantyhose sample and Figure 1 shows a schematic illustration of the pantyhose produced. The covering level of the single covered yarn and the draw ratio of the core yarn of sample $S$ are nearly equal to those of normal pantyhose currently on the market.

TABLE 1 Specification

\begin{tabular}{ccc}
\hline Sample & $\begin{array}{c}\text { Covering } \\
\text { level (tpm) }\end{array}$ & \multicolumn{1}{c}{ Region } \\
\hline$S$ & 1500 & Thigh - Ankle \\
& 3000 & --- \\
$S a$ & 1500 & Thigh - MCA \\
& 3000 & MCA - Ankle \\
$S c$ & 1500 & Thigh - Calf \\
& 3000 & Calf - Ankle \\
$S k$ & 1500 & Thigh - Knee \\
& 3000 & Knee - Ankle \\
\hline \multicolumn{3}{l}{ (MCA: the middle of calf and ankle) }
\end{tabular}

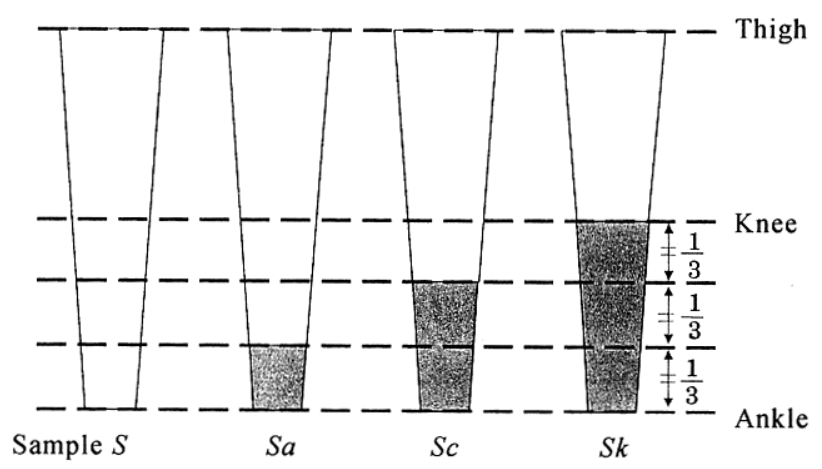

Figure 1 Pantyhose samples

(Covering level (tpm): 1500

\subsection{Sensory Test}

Table 2 lists the body dimensions of a Japanese female student who served as the subject for the wear experiment. Her measurements are approximately equal to those of a typical Japanese female [5]. The evaluators were six healthy female Japanese students, who are 22 24 years old. When the subject puts on the samples, we are careful that she fits her leg parts, i.e. thigh, knee, and ankle, to the corresponding parts of the samples. In a test of visual impressions, we use a paired comparison method (a modified version of the Scheffe-Nakaya) [11] and evaluate samples according to a five-criteria system with seven items listed in Table 3. For example, the five intensity scales for an item of "Light/Dark" are indicated by 1: "Moderately light", 2: "Slightly light", 3: "Neither light nor dark", 4: "Slightly dark", and 5: "Moderately dark".

Figure 2 shows a schematic illustration of the experimental conditions. A wear experiment to evaluate the visual impressions was carried out in a darkroom controlled at $25^{\circ} \mathrm{C}$ and $60 \%$ R.H. The light source $\left(\mathrm{D}_{65}\right)$ was a standard illuminant. The body of the subject except for the leg section was

TABle 2 Body dimensions of subject

\begin{tabular}{llll}
\hline \multicolumn{2}{c}{ Height $(\mathrm{cm})$} & \multicolumn{2}{c}{ Circumference $(\mathrm{cm})$} \\
\hline Crotch & 81.2 & Thigh & 55.0 \\
Mid-patellar & 46.2 & Knee & 33.6 \\
& & Calf & 34.1 \\
& & Ankle & 20.6 \\
\hline
\end{tabular}

TABLE 3 Evaluation items

Not sheer - Sheer

Doesn't look like bare skin - Looks like bare skin

Thick - Thin

Not lustrous - Lustrous

Coarse grain - Fine grain

Not beautiful - Beautiful

Dislike - Like 


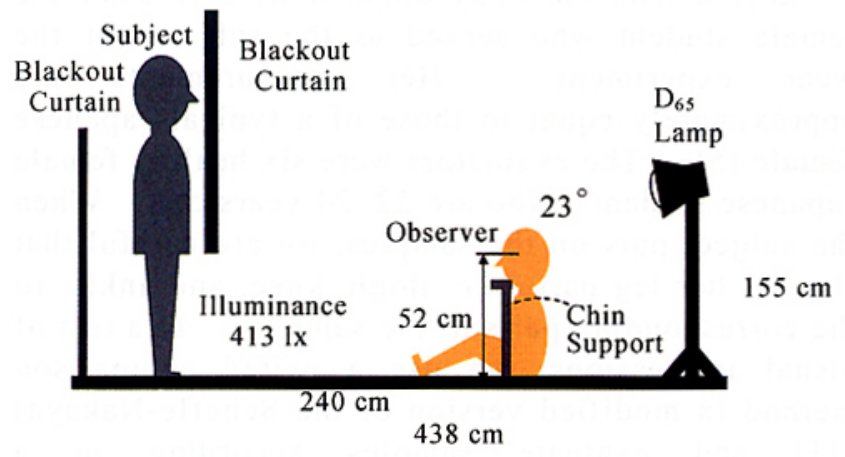

Figure 2 Experimental conditions (Side view)

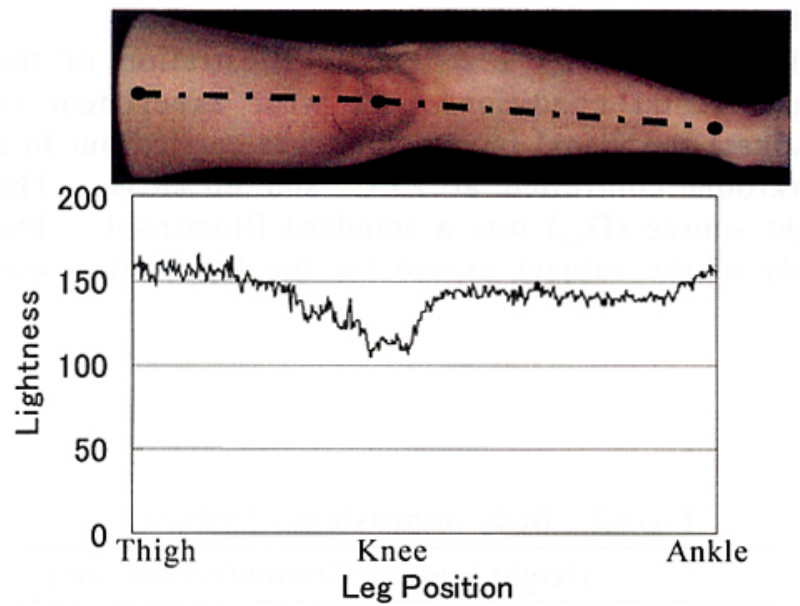

FIGURE 3 Lightness variation of bare skin leg along a centerline for image analysis

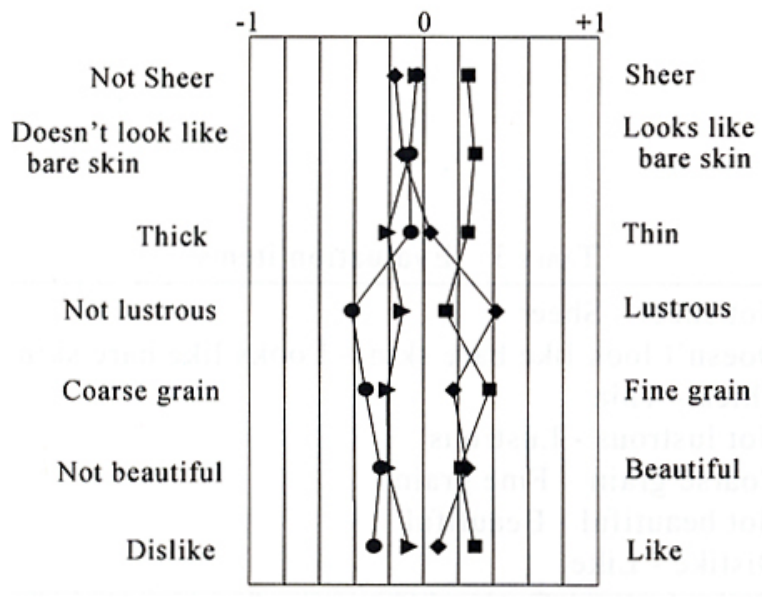

Figure 4 Visual impressions of samples

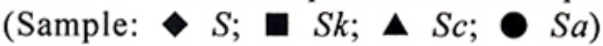

covered with a blackout curtain to eliminate possible effects of other visual information. The distance between the subject in standing position and the observer in sitting position was $240 \mathrm{~cm}$. The position of the object's eyes was fixed at a height of $52 \mathrm{~cm}$ from the ground using a chin support. The intensity of illumination from the light source was $413 \pm 15 \mathrm{~lx}$ near the legs of the subject.

\subsection{Image Analysis}

For the evaluation of visual information, photographs of the legs of the subject were taken from the front using a digital camera (Nikon model E3) fixed at a distance of $160 \mathrm{~cm}$ and a height of $52 \mathrm{~cm}$. Color images of the bare skin leg and the legs covered with the four kinds of samples were converted into step-luminance and lightness data [12]. In these color images, a variation of the lightness difference between the fronts of legs with and without the sample was calculated along a straight line drawn through the centers of the lower leg, i.e. the thigh, knee, and ankle of the left leg of the subject. The centerline located for this image analysis and the lightness variation of the bare skin leg are shown in Figure 3.

\section{Results and Discussion}

\subsection{Visual Impressions}

Figure 4 shows the profile of the visual impressions for pantyhose samples. In this evaluation, we assigned numerical values of $-2 \sim+2$ to a five-criteria system and the average preferences were calculated. Firstly, the sample $S$ was mostly described by both "Not Sheer" and "Lustrous". Secondly, the three kinds of samples with the covering level of the single covered yarns changed were mainly characterized as follows: (1) Sample $S a$ was considered "Not lustrous", "Coarse grain", "Not beautiful" and "Dislike"; (2) Sample $S c$ was regarded as "Thick" and "Not beautiful"; (3) And sample $S k$ was given "Sheer", "Looks like bare skin", "Thin", "Fine grain" and "Like". Sample $S a$ was characterized by the negative assessment of "Dislike". This may be explained by noting that as the visual impression is influenced by the evenness of sheerness over the entire leg, the smallest area with the covering level of 3,000 tpm is a very unique section, i.e. a step of unevenness in the entire leg. As a whole, the evaluation of sample $S k$ was more favorable than the others. Therefore, it was found that the aesthetics and sheerness of pantyhose are greatly influenced by changing the covering level of the single covered yarn in the leg parts. However, in a statistical analysis of difference between average preferences of the samples ( significance level $=0.15$ ), there observed differences in the item of "Like/Dislike" only without the 


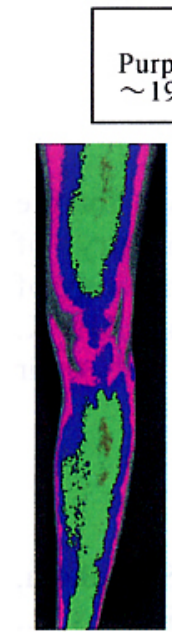

Bare skin

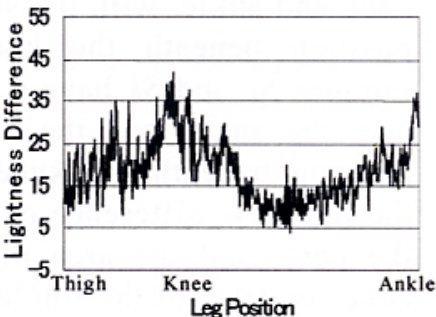

(2) Sample $S a$

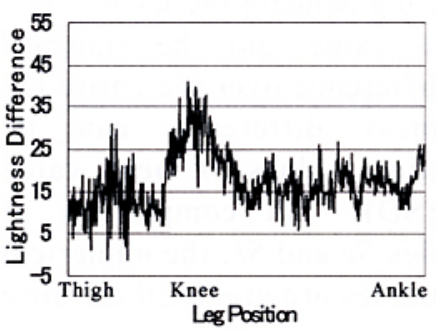

(3) Sample $S c$

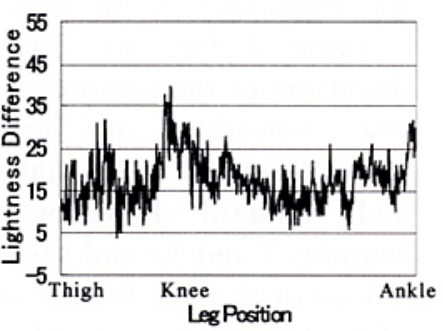

(4) Sample $S k$
FIGURE 7 Variation of lightness difference

TABLE 4 Mean and standard deviation of

\begin{tabular}{crrrr}
\hline & \multicolumn{4}{c}{ Sample } \\
& \multicolumn{1}{c}{$S$} & \multicolumn{1}{c}{$S a$} & $S c$ & \multicolumn{1}{c}{$S k$} \\
\hline Mean & 18.9 & 18.6 & 17.2 & 17.7 \\
SD & 7.6 & 7.3 & 6.6 & 5.7 \\
\hline
\end{tabular}

combination effect (or deviations from subtractivity). In addition, the other main effects and their combination effects were strongly influenced by the unique impression of sample $S a$.

\subsection{Image Analysis}

Figure 5 shows a representation of step-luminance on the image of the leg with and without the samples. In comparison with the bare skin leg, the leg covered by each sample, $S, S a, S c$, or $S k$, has a greater increase of luminance and/or lightness on the lower leg from knee to ankle. And the area of greater lightness with two ranges of $195 \sim 205$ and $205 \sim$ increases with
STEP-LUMINANCE

Purple: $135 \sim 155$, Blue: $155 \sim 175$, Green: 175 $\sim 195$, Brown: $195 \sim 05$, Red: $205 \sim$

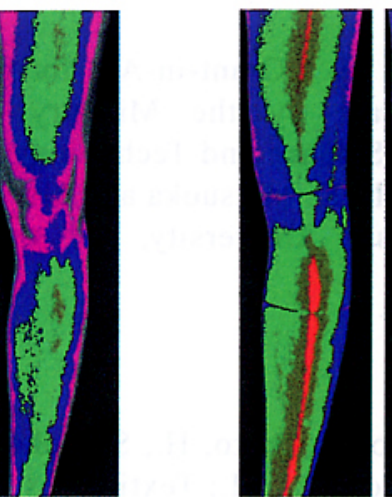

Sample: $S$

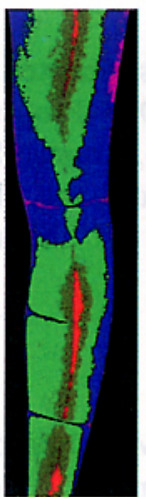

$\mathrm{Sa}$

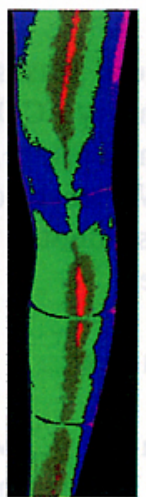

Sc
(1) Sample $S$

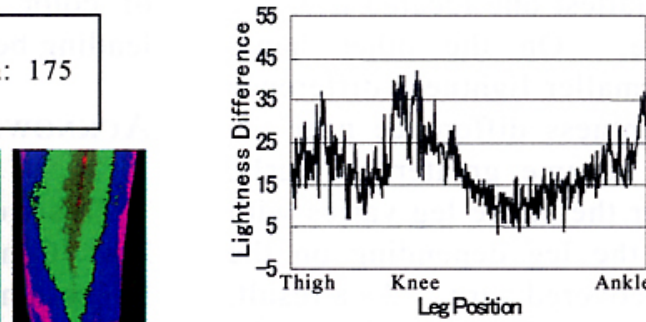
lightness difference

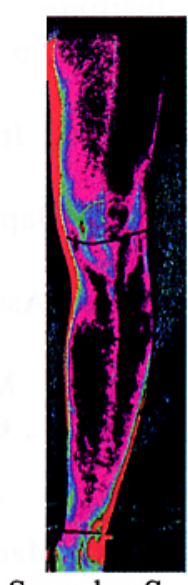

Figure 6
$\mathrm{Sa}$

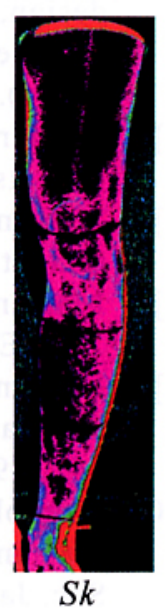

Sk
Lightness difference of samples 
have the two greatest lightness differences around the knee and ankle, with the smallest one located near the position beneath the knee. On the other hand, samples $S c$ and $S k$ have a smaller lightness difference near the ankle, and the lightness difference near the position beneath the knee became greater. Clearly, the lightness difference over the entire leg varies with the parts and the area of the leg depending on the covering level of the single covered yarn. As a result, the visual impressions of the pantyhose are affected by the variation of the lightness difference over the entire leg, especially on the lower leg beneath the knee.

Table 4 lists the mean value and the standard deviation of the lightness difference over the entire leg. The variation of lightness difference can be characterized by the statistical values of mean value and standard deviation (SD). In comparison of samples $S$ and $S a$ and samples $S c$ and $S k$, the numerical values of the two former samples are greater than those of the latter ones. Furthermore, in comparing samples $S c$ and $S k$, sample $S k$ has a greater mean value but has a smaller value of standard deviation. From this standpoint, there is a good agreement between the better evaluation from the visual impression and the smaller standard deviation of the lightness difference in the order of $S, S a, S c$, and $S k$. To improve the aesthetic appeal of pantyhose, therefore, it is important to decrease the mean value and the standard deviation on the variation of the lightness difference between the legs with and without the pantyhose and to calculate those values for developing aesthetic pantyhose. Furthermore, it appears that comfortable pantyhose can be produced using a hybrid knitting system.

\section{Conclusions}

To clarify the aesthetic properties of pantyhose, the design and development of a new product and a wear experiment were conducted. The results are shown as follows: (1) The aesthetic properties of pantyhose can be controlled by changing the covering level of the single covered yarn in the leg parts; (2) It is important to decrease the mean value and the standard deviation of the lightness difference between the legs with and without the pantyhose on the lower leg from knee to ankle; (3) A mechanical hybrid system combining both the covering and knitting processes is useful for improving the aesthetic properties and sheerness of pantyhose.

In a further study, we are planning to investigate a restriction on the lightness difference, and an insertion of color core yarn into a single covered yarn for lending beauty to a woman's leg.

\section{Acknowledgments}

This work was supported by a Grant-in-Aid for the 21 st Century COE Program by the Ministry of Education, Culture, Sports, Science and Technology of Japan. We wish to thank Mr. K. Matsuoka and Ms. K. Maeda, students of Shinshu University, for their assistance.

\section{References}

[1] Azuma, Y., Matsumoto, Y., Saito, H., Shinohara, K., Wakashima, K., Morooka, H.; Textile Res. J., 70, 763 (2000)

[2] Fujimoto, T.; Jpn. Res. Ass. Text. End-uses, 30, 80 (1989).

[3] Fujimoto, T.; Jpn. Res. Ass. Text. End-uses, 30, 118 (1989).

[4] Harada, T.; Jpn. Res. Ass. Text. End-uses, 36, 24 (1995).

[5] Human body dimensions data for ergonomic design, Report of National Institute of Bioscience and Human Technology, vol. 2, p.96 (1994).

[6] Inamura, A., Nakanishi, M. and Niwa, M.; Jpn. Res. Ass. Text. End-uses, 36, 109 (1995).

[7] Matsumoto, Y.; TOKUGAN 2003-117641, Japan (patent pending).

[8] Matsumoto, Y., and Morooka, H.; Jpn. Res. Assn. Text. End-Uses, 38, 711 (1997).

[9] Matsumoto, Y., Shinohara, K., Takatera, M., Azuma, Y., and Morooka, H.; Textile Res. J., 69, 641 (1999).

[10] Morooka, H., Wakashima, K., Azuma, Y., Matsumoto, Y., and Morooka, H.; J. Text. Mach. Soc. Japan, 52, 186 (1999).

[11] Research Committee of Sensory Evaluation, Union of Japanese Scientists and Engineers, "SENSORY EVALUATION HANDBOOK", JUSE Press Ltd., p.379 (1973).

[12] The Color Science Association of Japan, "Handbook of Color Science [Second Edition]", University of Tokyo Press, p.89 (1998). 